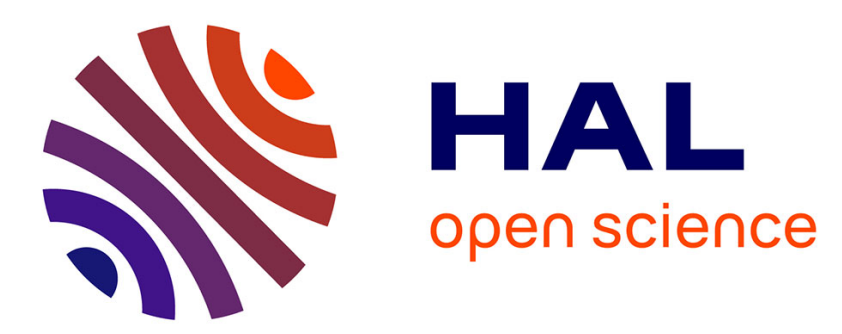

\title{
Continuous Wavelet Decompositions, Multiresolution, and Contrast Analysis
}

Marc Duval-Destin, Maria Amelia Muschietti, Bruno Torrésani

\section{To cite this version:}

Marc Duval-Destin, Maria Amelia Muschietti, Bruno Torrésani. Continuous Wavelet Decompositions, Multiresolution, and Contrast Analysis. SIAM Journal on Mathematical Analysis, 1993, 24 (3), pp.739-755. 10.1137/0524045 . hal-01279506

\section{HAL Id: hal-01279506 https://hal.science/hal-01279506}

Submitted on 26 Feb 2016

HAL is a multi-disciplinary open access archive for the deposit and dissemination of scientific research documents, whether they are published or not. The documents may come from teaching and research institutions in France or abroad, or from public or private research centers.
L'archive ouverte pluridisciplinaire HAL, est destinée au dépôt et à la diffusion de documents scientifiques de niveau recherche, publiés ou non, émanant des établissements d'enseignement et de recherche français ou étrangers, des laboratoires publics ou privés. 


\title{
CONTINUOUS WAVELET DECOMPOSITIONS, MULTIRESOLUTION AND CONTRAST ANALYSIS*
}

\author{
M. DUVAL-DESTIN ${ }^{1}$, M.A. MUSCHIETTI ${ }^{2}$ AND B. TORRESANI ${ }^{3}$
}

\begin{abstract}
A continuous version of multiresolution analysis is described, starting from usual continuous wavelet decompositions. Scale discretization leads to decompositions into functions of arbitrary bandwidth, satisfying QMF-like conditions. Finally, a nonlinear multiresolution scheme is described, providing multiplicative reconstruction formulas.
\end{abstract}

Key words. Wavelets, Multiresolution analysis, Contrast analysis

Wavelet analysis of a function $f \in L^{2}(\mathbb{R})$ basically consists in the decomposition of $f$ as a sum of wavelets $\psi_{(b, a)}(x)=\frac{1}{a} \psi\left(\frac{x-b}{a}\right)$, dilated and translated copies of the mother wavelet $\psi$, an integrable function with vanishing integral. The coefficients of the decomposition are nothing but the scalar products $\left\langle f, \psi_{(b, a)}>\right.$.

Continuous wavelet decompositions have been introduced (or re-introduced, since similar tools have been used by mathematicians for a long time to study certain classes of operators [Cal]) by A. Grossmann and J. Morlet [Gr-Mo], and many applications have been developed (see [Co-Gr-Tc] and references therein), in particular in a signal analysis context [Gr-KM-Mo] [De-E-Gu-KM-Tc-To]. The discretization of the continuous formulas was studied later [Da1], and handled by the introducion of the notion of frames of wavelets (see also [He-Wa]). Continuous wavelets and frames of wavelets were used later on for many problems of applied mathematics and physics, and in particular to model physiology of vision, following the program of D. Marr [Mar] (see also $[\mathrm{Fr}-\mathrm{Mo}],[\mathrm{DD}])$. In particular, they allowed one of us to introduce the notion of scale-space contrast $[\mathrm{DD}]$, and to exhibit multiplicative reconstruction formulas from the contrast functions.

The discovery of orthonormal bases of wavelets by J.O. Stromberg [Str] and Y. Meyer and his collaborators (see [Me], and references therein) opened a new door in the understanding of the theory, making in particular the connection with sub-band coding, familiar to electrical engineers and image processers [Da2]. This moreover led to the construction of a large family (a library in the author's terminology) of orthonormal bases, called wavelet packets bases, providing adaptive decompositions of functions directly implementable in fast (pyramidal) algorithms [Co-Me-Qu-Wi]. A continuous analogue of wavelet packets was proposed in [To1][To2], in which the pyramidal algorithmic structure is unfortunately lost. The key point of the construction of wavelet packets is that they allow to control the relative bandwidth of the analyzing functions in the Fourier space (the so-called surtension factor), as a function of frequency.

We present here a general construction, allowing the construction of wavelet packets starting from an usual continuous wavelet analysis. By wavelet packets we mean

\footnotetext{
*Received by the editors September 1991.

*CERMA/DEA, Etablissement technique d'Angers, BP 4107, 49041 Angers cedex, France.

** Departamento de Matemáticas, Universidad de La Plata, C.C. 172, 1900 La Plata, Argentina.

${ }^{* * *}$ CPT, CNRS-Luminy, Case 907, 13288 Marseille Cedex 09, France.
} 
families of functions generated from a single one by simple transformations and whose relative bandwidth is non-constant (contrary to usual wavelets) and can be matched to a given analyzed funtion. Let us stress here that our construction is not a continuous version of that of [Co.Me.Qu.Wi], and is actually quite different. Starting from an admissible analyzing wavelet $\psi$, it is well known that one can associate with it a scaling function $\phi$, and then mimic the multiresolution construction. This is briefly described in section 1 . In a similar way, the continuous decomposition over scales can be replaced by a discrete one, as shown in section 2 . The corresponding sequence of scale parameters can be chosen arbitrarily, and the functions appearing in the decomposition (the wavelet packets) must be matched to that sequence. As a particular case, a geometric sequence yields usual Littlewood-Paley decompositions. In section 3, we continue developing the analogy between continuous wavelet analysis and multiresolution analysis. In particular, the scaling function can be expressed as a continuous infinite product of dilated copies of a low-pass filter, denoted by $\mu_{0}$ (let us recall that a continuous product, or multiplicative integral $\prod_{a}^{b}(d \mu(x)) f(x)$ is defined to equal $\exp \left[\int_{a}^{b} \ln f(x) d \mu(x)\right]$ whenever such an expression makes sense [Gui]). Then using the wavelet packets construction, a continuous finite product of such $\mu_{0}$ filters yields new low-pass filters, denoted by $m_{0}$, which makes the connection with the quadrature mirror filters (QMFs) appearing in multiresolution analysis. Section 4 is devoted to the construction of contrast analysis. We define the infinitesimal contrast function $c_{a}(x)$ of $f(x)$ as (roughly speaking) the quotient of the details of $f(x)$ at scale $a$ by the approximation of $f(x)$ at scale $a$. Then under some analyticity assumptions on $|\hat{\phi}|^{2}$, we show that such contrast functions provide a multiplicative decomposition of $f(x)$ over scales. Otherwise stated $f(x)$ can be continuously factorized into exponentials of contrast functions. Such continuous product formulas can be discretized in the same way than in section 3 , yielding very simple factorization formulas, still interpretable in terms of the integrated contrast functions introduced in [DD]. This procedure is closely connected to models of human vision [DD],[Me-DD-Ge] in which the emphasis is put on multiresolution image processing and logarithmic light sensitivity.

\section{INFINITESIMAL MULTIRESOLUTION ANALYSIS OF $L^{2}(\mathbb{R})$.}

\section{1-1. Bilinear analysis.}

Let us start from standard notions of continuous wavelet analysis (Throughout this paper, our conventions for Hermitian product and Fourier transform in $L^{2}(\mathbb{R})$ are the following ones. $<f, g\rangle=\int f(x) g(x) * d x$, where the star denotes complex conjugation, and $\hat{f}(\xi)=<f, \epsilon_{\xi}>$ where $\left.\epsilon_{\xi}(x)=\exp (i \xi x)\right)$ We will focus on the analysis of $L^{2}(\mathbb{R})$, and sometimes describe in a few words the corresponding results in the $H^{2}(\mathbb{R})$ context (we will denote here by $H^{2}(\mathbb{R})=\left\{f \in L^{2}(\mathbb{R}), \hat{f}(\xi)=0 \forall \xi \leq 0\right\}$ the complex Hardy space).

Generically, a wavelet (or mother wavelet) is a function $\psi \in L^{1}(\mathbb{R})$ such that the following admissibility condition holds:

$$
c_{\psi}=\int_{0}^{\infty}|\hat{\psi}(u)|^{2} \frac{d u}{u}=\int_{0}^{\infty}|\hat{\psi}(-u)|^{2} \frac{d u}{u}=1
$$


If $\hat{\psi}$ is differentiable, $(1-1)$ basically means that $\hat{\psi}(0)=0$, otherwise stated:

$$
\int_{-\infty}^{\infty} \psi(x) d x=0
$$

Such a mother wavelet provides the following analysis of $L^{2}(\mathbb{R})$ : for any $(b, a) \in$ $\mathbb{R} \times \mathbb{R}_{+}^{*}$, one introduces the wavelet:

$$
\psi_{(b, a)}(x)=\frac{1}{a} \psi\left(\frac{x-b}{a}\right)
$$

and one has the following

THEOREM 1. Let $\psi$ be a mother wavelet. Then any $f \in L^{2}(\mathbb{R})$ decomposes as follows:

$$
f=\int_{\mathbb{R} \times \mathbb{R}_{+}^{*}}<f, \psi_{(b, a)}>\psi_{(b, a)} \frac{d b d a}{a}
$$

strongly in $L^{2}(\mathbb{R})$.

Proof. The proof follows from standard arguments, and we sketch it for completeness. Let

$$
T_{f}(b, a)=<f, \psi_{(b, a)}>\text {. }
$$

Then $T_{f} \in L^{2}(\mathbb{R}, d b)$ by Young's convolution inequality. Define

$$
d_{a}(x)=\int_{\mathbb{R}} T_{f}(b, a) \psi_{(b, a)}(x) d b
$$

Again, Young's inequality ensures that $d_{a} \in L^{2}(\mathbb{R})$ for any $a \in \mathbb{R}_{+}^{*}$. Moreover, setting:

$$
s_{\varepsilon}^{\rho}(x)=\int_{\varepsilon}^{\rho} d_{a}(x) \frac{d a}{a},
$$

one has that $s_{\varepsilon}^{\rho} \in L^{2}(\mathbb{R})$ and $\left|\hat{s_{\varepsilon}}(\xi)\right|<|\hat{f}(\xi)|$ a.e., so that the Lebesgue dominated convergence theorem implies that

$$
\lim _{\varepsilon \rightarrow 0, \rho \rightarrow \infty}\left\|f-s_{\varepsilon}^{\rho}\right\|_{2}=0
$$

yielding the theorem.

Theorem 1 has been known for a long time by mathematicians as Calderón's identity [Cal], [Fr-Ja-We]. It was rediscovered more recently in a signal analysis context by Grossmann and Morlet [Gr-Mo], and interpreted as follows: $T_{f} \in L^{2}\left(\mathbb{R} \times \mathbb{R}_{+}^{*}\right)$ is called the wavelet transform of $f$ with respect to the analyzing wavelet $\psi$. If $\psi$ is sufficiently well localized in time and frequency (i.e. both $\psi$ and $\hat{\psi}$ have sufficient decay at infinity), $T_{f}$ gives informations on the time-frequency localization of $f$. Conversely, $(1-3)$ states that the wavelet transform is invertible on its range, allowing the reconstruction of the analyzed function from its wavelet transform.

If one restricts to the Hardy space $H^{2}(\mathbb{R})$, a weaker admissibility condition (concerning only the positive frequency part of $\psi$ ) is sufficient. Simply assuming that:

$$
c_{\psi}=\int_{0}^{\infty}|\hat{\psi}(u)|^{2} \frac{d u}{u}=1
$$


theorem 1 holds for any $f \in H^{2}(I R)$.

We will need in the sequel a somewhat stronger assumption. We will call $\psi$ an infinitesimal wavelet if $\psi$ is a real-valued wavelet, belonging to the atomic Hardy space $H_{a}^{1}(\mathbb{R}) . H_{a}^{1}(\mathbb{R})$ is the space of the functions of $L^{1}(\mathbb{R})$ such that their Hilbert transform (We recall that the Hilbert transform $H$. $f$ of a function $f$ can be defined in the Fourier space as $\widehat{H} \cdot f(\xi)=-i \operatorname{sgn}(\xi) \hat{f}(\xi))$ is also in $L^{1}(\mathbb{R})$ (see [Co-We] for a detailed account of the theory of real Hardy spaces).

Let then $\psi(x)$ be an infinitesimal wavelet, and let $q(x)$ be its autocorrelation function:

$$
q(x)=\int_{-\infty}^{\infty} \psi(y) \psi(x+y) d y .
$$

Clearly $q \in H_{a}^{1}(\mathbb{R})$, and one easily checks that

$$
\int_{0}^{\infty} q(x) d x=\int_{-\infty}^{0} q(x) d x=0 .
$$

Let

$$
\begin{gathered}
p(x)=\frac{1}{x} \int_{0}^{x} q(y) d y, \quad x>0 \\
=-\frac{1}{x} \int_{x}^{0} q(y) d y, \quad x<0
\end{gathered}
$$

denote the mean function of $q(x)$. One also has:

$$
\hat{p}(\xi)=\int_{1}^{\infty}|\hat{\psi}(t \xi)|^{2} \frac{d t}{t} .
$$

We will also denote $p_{a}(x)=\frac{1}{a} p\left(\frac{x}{a}\right)$.

LEMMA. $p \in L^{1}(\mathbb{R})$.

Proof. Let $q^{+}$(resp. $q^{-}$) denote the restriction of $q$ to the positive (resp. negative) real axis. Since $q^{+} \in H_{a}^{1}(\mathbb{R}), q^{+}(x)$ admits an atomic decomposition with $(1, \infty)$ atoms:

$$
q^{+}(x)=\sum_{n} \lambda_{n}^{+} a_{n}^{+}(x)
$$

where

$$
\sum_{n}\left|\lambda_{n}^{+}\right|<\infty
$$

and the $(1, \infty)$-atoms $a_{n}^{+}(x)$ are compactly supported $L^{1}$-functions with support in an interval $I_{n}^{+}$, such that

$$
\int a_{n}^{+}(x) d x=0
$$

and

$$
\left\|a_{n}^{+}\right\|_{\infty} \leq \frac{1}{\left|I_{n}^{+}\right|} .
$$

Moreover, such a decomposition is not unique, and (because of $(1-8)$ ) can be chosen in such a way that $I_{n}^{+} \subset \mathbb{R}^{+}$. Indeed, if $q^{+}(x)=\sum_{n} \lambda_{n}^{+} a_{n}^{+}(x)$ is an atomic 
decomposition of $q^{+}(x)$ with $(1, \infty)$-atoms $a_{n}^{+}(x)$, then it also admits an atomic decomposition $q^{+}(x)=\sum_{n} \lambda_{n}^{+} b_{n}^{+}(x)$, where $b_{n}^{+}(x)=\left[a_{n}^{+}(x)+a_{n}^{+}(-x)\right] \chi^{+}(x)$ are also $(1, \infty)$-atoms, supported on the positive real axis (here $\chi^{+}(x)$ is the Heaviside step function). A similar property obviously holds for $q^{-}$. We then assume from now on that $\operatorname{Supp}\left(a_{n}^{ \pm}\right) \subset \mathbb{R}^{ \pm}$. Denote $p_{n}^{+}(x)=\frac{1}{x} \int_{0}^{x} a_{n}^{+} d y(y)$ and $p_{n}^{-}(x)=-\frac{1}{x} \int_{x}^{0} a_{n}^{-}(y) d y$. Then

$$
\|p\|_{1} \leq \sum_{n}\left(\left|\lambda_{n}^{+}\right|\left\|p_{n}^{+}\right\|_{1}+\left|\lambda_{n}^{-}\right|\left\|p_{n}^{-}\right\|_{1}\right)
$$

so that $\|p\|_{1}<\infty$ if the $\left\|p_{n}^{ \pm}\right\|_{1}$ are uniformly bounded. Now consider for instance $p_{n}^{+}$, and let $I_{n}^{+}=[a, b]$. Then,

$$
\begin{aligned}
\left\|p_{n}\right\|_{1} \leq & \frac{1}{b-a}\left[\int_{a}^{\frac{a+b}{2}} \frac{x-a}{x} d x+\int_{\frac{a+b}{2}}^{b} \frac{b-x}{x} d x\right] \\
& \leq \frac{1}{b-a}\left[a \ln \frac{2 a}{a+b}+b \ln \frac{2 b}{a+b}\right]
\end{aligned}
$$

which yields the desired result. The lemma is then proved.

Then $0 \leq \hat{p}(\xi)<\infty$. Let $\phi$ be such that:

$$
\hat{p}(\xi)=|\hat{\phi}(\xi)|^{2}=\int_{|\xi|}^{\infty}|\hat{\psi}(u \operatorname{sgn}(\xi))|^{2} \frac{d u}{u} .
$$

In other words, $|\hat{\psi}(u \xi)|^{2}=-u \partial_{u}|\hat{\phi}(u \xi)|^{2}$ for all $\xi \in \mathbb{R}$, and $\lim _{\xi \rightarrow \infty}|\hat{\phi}(\xi)|^{2}=0 . \quad \phi$ is called a scaling function, and one associates to it the corresponding:

$$
\phi_{(b, a)}(x)=\frac{1}{a} \phi\left(\frac{x-b}{a}\right) .
$$

We will see in section 3 that $\phi(x)$ satisfies some kind of scaling equation (see eq. (3$7)$ ), and that infinite product formulas for $\hat{\phi}(\xi)$ can be obtained (eq. (3-5)). Clearly, $(1-14)$ does not characterize $\phi$; althouh it is in general unnecessary, one can always restrict to a real-valued $\hat{\phi}$. Notice that the squared modulus of the Fourier transform of the scaling function is by construction a decreasing (resp. increasing) function for positive (resp. negative) values of $\xi$.

To any $f \in L^{2}(\mathbb{R})$ associate:

$$
s_{a}(x)=\int_{\mathbb{R}}<f, \phi_{(b, a)}>\phi_{(b, a)}(x) d b
$$

that is:

$$
s_{a}(x)=\int_{a}^{\infty} d_{u}(x) \frac{d u}{u}=\left(f * p_{a}\right)(x) .
$$

Then $s_{a} \in L^{2}(\mathbb{R})$, and one has the following decompositions, whose proofs are immediate from that of theorem 1 :

COROLlary. Let $\psi$ an infinitesimal wavelet, and $\phi$ an associated scaling function. Then any $f \in L^{2}(\mathbb{R})$ can be expressed as:

$$
f=\lim _{a \rightarrow 0} s_{a}
$$




$$
=s_{a_{0}}+\int_{0}^{a_{0}} d_{a} \frac{d a}{a}
$$

strongly in $L^{2}(\mathbb{R})$.

The corollary also holds in the $H^{2}(\mathbb{R})$ context.

In terms of linear filtering, $\phi$ can be seen as a low-pass filter, and $\psi$ as a band-pass filter. Indeed, it is usual to consider analyzing wavelets such that $\hat{\psi}(\xi)$ is well localized in the Fourier domain around some frequeny $\omega_{0}$. Then $\hat{\psi}(t \xi)$ is localized around $\xi \cong \omega_{0} / t$, and $\hat{p}(\xi)$ (and $\hat{\phi}(\xi)$ ), built by "gluing together the $|\hat{\psi}(t \xi)|^{2}, t \in[1, \infty[$ " is centered on the zero frequency. Then $s_{a}$ describes the low-frequency content of $f$ up to the scale $a$ (otherwise stated the approximation at scale $a$ ), and $d_{a}$ describes the content of $f$ around the scale $a$ (i.e. the details at scale $a$ ).

Denote now by $Q_{a}$ and $P_{a}$ the convolution operators, defined respectively by the multiplyiers $\hat{q}_{a}(\xi)=|\hat{\psi}(a \xi)|^{2}$ and $\hat{p}_{a}(\xi)=|\hat{\phi}(a \xi)|^{2}$ :

$$
\begin{aligned}
& {\left[\widehat{Q_{a} f}\right](\xi)=\hat{q}_{a}(\xi) \hat{f}(\xi)=\hat{d}_{a}(\xi)} \\
& {\left[\widehat{P_{a} f}\right](\xi)=\hat{p}_{a}(\xi) \hat{f}(\xi)=\hat{s}_{a}(\xi)}
\end{aligned}
$$

The previous corollary then yields an approximation of the identity by the operators $Q_{a}$ and $P_{a}$. If one introduces the spaces:

$$
V_{(a)}=P_{a} \cdot L^{2}(\mathbb{R})
$$

then it clearly follows from the monotonicity of $|\hat{\phi}|$ that for any $a<a^{\prime}, V_{\left(a^{\prime}\right)} \subseteq V_{(a)}$. By analogy with the usual multiresolution analysis [Me], we call such a collection of spaces a continuous (or infinitesimal) multiresolution analysis. In particular, it must be remarked that $V_{(a)}$ increases to the whole $L^{2}(I R)$ as $a$ goes to 0 and decreases to $\{0\}$ as $a$ goes to $\infty$. Notice that all the $V_{(a)}$ spaces are translation invariant; then they are not closed, except in the case where $|\hat{\phi}|=\chi_{\Omega} \rho$ where $\chi_{\Omega}$ is the characteristic function of some measurable set $\Omega$ and $\rho$ a bounded strictly positive function (see e.g. $[\mathrm{Ru}])$.

\section{1-2. Linear analysis.}

It is well known that the reconstructing and the analyzing wavelets can be decoupled. Otherwise stated one can use differents infinitesimal wavelets for the computation of the coefficients and for the synthesis of the analyzed function from the coefficients (see [Ho-Tc], [Ho] for beautiful applications of this property in different contexts). In such a case, the admissibility condition $(1-1)$ has to be modified accordingly [Ho-Tc].

A particular example of such a decoupling, which has been known for a long time, consists in taking formally a Dirac distribution for the reconstructing wavelet. Assuming instead of $(1-1)$ that

$$
k_{\psi}=\int_{0}^{\infty} \hat{\psi}(u) \frac{d u}{u}=\int_{0}^{\infty} \hat{\psi}(-u) \frac{d u}{u}=1,
$$


one has the following decomposition of any $f \in L^{2}(\mathbb{R})$ :

$$
f(x)=\int_{\mathbb{R}_{+}^{*}}<f, \psi_{(x, a)}>\frac{d a}{a}
$$

strongly in $L^{2}(\mathbb{R})$. This is the so-called Morlet reconstruction formula of $f$ from its wavelet coefficients. Such a linear analysis (linear in the $\psi$ function) generates a continuous multiresolution analysis as follows: introduce the linear scaling function $\varphi \in L^{1}(\mathbb{R})$, defined by:

$$
\hat{\varphi}(\xi)=\int_{|\xi|}^{\infty} \hat{\psi}(u \operatorname{sgn}(\xi)) \frac{d u}{u} .
$$

$\varphi$ is also such that $\hat{\psi}(u \xi)=-u \partial_{u} \hat{\varphi}(u \xi)$ for all $\xi \in \mathbb{R}$. Associate to $\varphi$ the following functions:

$$
\varphi_{(b, a)}(x)=\frac{1}{a} \varphi\left(\frac{x-b}{a}\right) .
$$

Finally introduce:

$$
\delta_{a}(x)=T_{f}(x, a)=<f, \psi_{(x, a)}>
$$

and

$$
\sigma_{a}(x)=<f, \varphi_{(x, a)}>.
$$

One then has the linear analogue of Theorem 1 and the corresponding corollary:

THEOREM 1'.

Let $\psi \in H_{a}^{1}(\mathbb{R})$ be a mother wavelet, such that $(1-21)$ holds, and let $\varphi$ be the associated linear scaling function. Then any $f \in L^{2}(\mathbb{R})$ can be decomposed as:

$$
\begin{array}{cc}
f=\lim _{a \rightarrow 0} \sigma_{a} & (1-27) \\
=\sigma_{a_{0}}+\int_{0}^{a_{0}} \delta_{a} \frac{d a}{a} \quad, a_{0} \in \mathbb{R}_{+}^{*} & (1-28) \\
=\int_{0}^{\infty} \delta_{a} \frac{d a}{a} & (1-29)
\end{array}
$$

strongly in $L^{2}(\mathbb{R})$.

\section{1-3. Comments and examples.}

The bilinear analysis gives the convenient scheme for the construction of orthonormal bases of wavelets [Me], [Da2]. Moreover, it is better adapted for the characterization of functional spaces (see e.g. [Fr.Ja.We], [Ho.Tc]). On the other hand, the linear analysis is very often used for signal analysis [Gr-KM-Mo], since it produces a simplified reconstruction formula, involving a one-dimensional integral.

There are many examples of admissible infinitesimal wavelets, some of which are described in [Gr-Mo] and [Gr-KM-Mo]. Actually, the simplest one arises quite naturally from infinitesimal multiresolution analysis. Indeed, let us take a Gaussian scaling function:

$$
\phi(x)=\frac{1}{\sqrt{\pi}} e^{-x^{2}}
$$


where the normalization constant that is fixed so that $(1-1)$ holds. Then in the bilinear analysis scheme, the infinitesimal wavelets can be deduced from $(1-7)$, and one can choose:

$$
\psi(x)=\frac{2 x}{\sqrt{\pi}} e^{-x^{2}}
$$

i.e. a derivative of Gaussian. The case of linear analysis yields a more famous infinitesimal wavelet. Taking for $\varphi$ a Gaussian function leads to the celebrated laplacian of Gaussian, used for a long time in image analysis and processing, and vision (see e.g. [DD],[Mar]):

$$
\psi(x)=\frac{1}{\sqrt{\pi}}\left(1-2 x^{2}\right) e^{-x^{2}} .
$$

In the next section, we will see how such derivatives of Gaussians simply lead to differences of Gaussians, that are also used in image theory and vision.

\section{WAVELET PACKETS IN $L^{2}(\mathbb{R})$.}

An important problem is that of the discretization of the continuous formulas derived in section 1. At least for numerical applications, one wants to be able to get discrete approximations of the identity in $L^{2}(I R)$, and to control the discretization error. Such a problem was handled in [Da1], where the author developed the theory of frames of wavelets. In particular, focusing on the scale discretization problem, if $\Lambda=\left\{a_{0} \lambda_{0}^{n}, n \in \mathbb{Z}\right\}$ denotes a geometric sequence in $\mathbb{R}$, for some positive $\lambda_{0}$, then $\sum_{\lambda \in \Lambda} Q_{\lambda}$ defines a linear operator, which is positive, bounded and invertible with bounded inverse for a suitable choice of the $\lambda_{0}$ parameter and the infinitesimal wavelet $\psi$. Moreover, this operator can often be written as a small perturbation of the identity (up to some multiplicative constant), which permits to consider the discretization as a good approximation of the continuous formula.

We are interested here in another way of discretizing the scales, such that the discretization errors are avoided. Such a procedure canonically produces new waveforms, that we will call wavelet packets. Start from a strictly decreasing sequence of positive real numbers:

$$
\ldots<a_{j+1}<a_{j}<a_{j-1}<\ldots
$$

such that $\lim _{j \rightarrow-\infty} a_{j}=\infty$ and $\lim _{j \rightarrow+\infty} a_{j}=0$.

Set:

$$
D_{j}(x)=\int_{a_{j+1}}^{a_{j}} d_{a}(x) \frac{d a}{a} .
$$

Then $D_{j} \in L^{2}(\mathbb{R})$, and

$$
\widehat{D_{j}}(\xi)=\hat{f}(\xi) \int_{a_{j+1}}^{a_{j}}|\hat{\psi}(a \xi)|^{2} \frac{d a}{a} \text { a.e. }
$$

Introducing the function $\Psi^{j}$, such that:

$$
\left|\widehat{\Psi^{j}}(\xi)\right|^{2}=\int_{a_{j+1}}^{a_{j}}|\hat{\psi}(a \xi)|^{2} \frac{d a}{a}
$$


one then has:

$$
\widehat{D_{j}}(\xi)=\hat{f}(\xi)\left|\widehat{\Psi^{j}}(\xi)\right|^{2}
$$

We will refer to the $\Psi^{j}$ functions as wavelet packets, since they are built up by gluing wavelets together (in the Fourier space, contrary to the case of those described in $[\mathrm{Co}-\mathrm{Me}-\mathrm{Qu}-\mathrm{Wi}])$. Such wavelet packets are also intuitively close to the atoms introduced in Littlewood-Paley theory (see e.g. [Fr-Ja-We]), obtained via a segmentation of Calderón's formula $(1-3)$ into integrals over dyadic cubes. In our case, the wavelet packets are obtained by a segmentation of $(1-3)$ into integrals over strips in the time-scale plane. The result is then that the atoms are generated in a simple way. Notice that $(2-3)$ does not completely define the wavelet packets. Once again, one can restrict to wavelet packets with positive-valued Fourier transform, but this is not necessary. By construction, the wavelet packets lead to a partition of unity in the Fourier space as follows:

$$
\sum_{j=-\infty}^{+\infty}\left|\widehat{\Psi^{j}}(\xi)\right|^{2}=\left|\widehat{\Phi^{j}}(\xi)\right|^{2}+\sum_{j=j_{0}}^{\infty}\left|\widehat{\Psi^{j}}(\xi)\right|^{2}=1
$$

for all $\xi \in \mathbb{R}$, where we have set:

$$
\widehat{\Phi^{j}}(\xi)=\hat{\phi}\left(a_{j} \xi\right)
$$

Defining the translates of the $\Phi^{j}$ and $\Psi^{j}$ as $\Phi_{b}^{j}(x)=\Phi^{j}(x-b)$ and $\Psi_{b}^{j}(x)=\Psi^{j}(x-b)$, we then have:

\section{THEOREM 2.}

Let $\psi$ an infinitesimal wavelet, and let $\Psi^{j}$ and $\Phi^{j}$ be associated wavelet packets and scaling functions as in $(2-3)$ and $(2-6)$. Then any $f \in L^{2}(\mathbb{R})$ can be decomposed as:

$$
f=\int_{\mathbb{R}}<f, \Phi_{b}^{j_{0}}>\Phi_{b}^{j_{0}} d b+\sum_{j=j_{0}}^{\infty} \int_{\mathbb{R}}<f, \Psi_{b}^{j}>\Psi_{b}^{j} d b
$$

strongly in $L^{2}(I R)$.

Denote by $\mathbf{Q}_{j}$ the convolution operator, defined by the multiplier $\left|\widehat{\Psi}^{j}(\xi)\right|^{2}$, and set $\mathbf{P}_{j}=P_{a_{j}}$. Let $V_{j}$ and $W_{j}$ denote respectively the images of $L^{2}(\mathbb{R})$ by $\mathbf{P}_{j}$ and $\mathbf{Q}_{j}$. This provides the following resolution of $L^{2}(I R)$ :

$$
\ldots \subset V_{j-1} \subset V_{j} \subset V_{j+1} \subset \ldots
$$

Again, it must be remarked that $V_{j}$ increases to the whole $L^{2}(\mathbb{R})$ as $j$ goes to $\infty$ and decreases to $\{0\}$ as $j$ goes to $-\infty$. Moreover, $V_{j}+W_{j}=V_{j+1}$, but in general $V_{i} \not \perp W_{i}$.

Remarks.

- The wavelet packets have vanishing integral by construction. Moreover, they can be seen as differences of two low-pass filters. Indeed, one has:

$$
\left|\widehat{\Psi^{j}}(\xi)\right|^{2}=\left|\widehat{\Phi^{j+1}}(\xi)\right|^{2}-\left|\widehat{\Phi^{j}}(\xi)\right|^{2}
$$


and the partial reconstructions

$$
D^{j}(x)=\int_{\mathbb{R}}<f, \Psi_{b}^{j}>\Psi_{b}^{j}(x) d b
$$

as differences of two smoothings of $f(x)$ at two consecutive scales:

$$
D^{j}(x)=S^{j+1}(x)-S^{j}(x)
$$

where

$$
S^{j}(x)=\int_{\mathbb{R}}<f, \Phi_{b}^{j}>\Phi_{b}^{j}(x) d b .
$$

Once more, we are close to the idea of "difference of two smoothings" wavelet familiar in vision theory.

- The same can be done in the linear analysis scheme. The wavelet packets are then defined as:

$$
\widehat{\Theta^{j}}(\xi)=\int_{a_{j+1}}^{a_{j}} \hat{\psi}(a \xi) \frac{d a}{a}
$$

and yield a partition of unity in the Fourier space:

$$
\sum_{j=-\infty}^{+\infty} \widehat{\Theta^{j}}(\xi)=\hat{\varphi}\left(a_{j_{0}} \xi\right)+\sum_{j=j_{0}}^{\infty} \widehat{\Theta^{j}}(\xi)=1
$$

The linear wavelet packets still appear as differences of smoothings at two consecutive scales, as:

$$
\widehat{\Theta^{j}}(\xi)=\hat{\varphi}\left(a_{j+1} \xi\right)-\hat{\varphi}\left(a_{j} \xi\right)
$$

and every $f \in L^{2}(\mathbb{R})$ decomposes as:

$$
f(x)=\sum_{j=-\infty}^{\infty}<f, \Theta_{x}^{j}>
$$

where $\Theta_{b}^{j}(x)=\Theta^{j}(x-b)$.

- The Gaussian example is once more very interesting, since it provides directly the DOGs, i.e. the differences of Gaussians at two different scales (see e.g. [Mar]).

- Consider the particular case where all the scale parameters $a_{j}$ are generated from a unique one $a_{0}$ as a geometric sequence:

$$
a_{j}=a_{0} \lambda^{-j}
$$

for some positive real number $\lambda>1$. Then the wavelet packets $\Psi^{j}$ (and also the $\Theta^{j}$ ) can be considered as wavelets in the usual sense, since they are generated from a unique function $\Psi^{0}$ (or $\Theta^{0}$ ) by dilations by powers of $\lambda$. In particular, the bilinear analysis yields the usual Littlewood-Paley analysis (also called dyadic wavelet analysis in $[\mathrm{Ma}])$.

- Let us finally stress that although we have chosen to use the term "Wavelet Packets", our construction is completely different than the wavelet packets construction proposed in [Co-Me-Qu-Wi]. Both constructions share the fact that the corresponding functions are of variable bandwidth, and that the bandwidth can be matched to a function to be analyzed. 


\section{MIRROR FILTERS AND FACTORIZABLE WAVELET PACK-} ETS.

\section{3-1. Mirror filters.}

The orthonormal bases of wavelet packets described for example in [Co-Me-QuWi] are derived in a very simple algorithmic way from the so-called Quadrature Mirror Filters, canonically associated with the multiresolution analysis. We will see in this section how infinitesimal multiresolution analysis can produce continuous versions of such filters, and how these infinitesimal filters can be put together to form QMFs for the wavelet packets. Let us start from the bilinear infinitesimal multiresolution analysis (the same arguments can be developed in the linear analysis scheme too), with scaling function $\phi$. If $|\hat{\phi}(a \xi)| \neq 0$, it follows from the monotonicity property of $|\hat{\phi}|$ that a determination of the complex logarithm $\ln \hat{\phi}(u \xi)$ can always be chosen for $0 \leq u \leq a$, so that the function

$$
\nu_{0}(a \xi)=a \partial_{a} \ln \hat{\phi}(a \xi) \quad \forall a, \xi \quad \text { s.t. } \hat{\phi}(a \xi) \neq 0
$$

can be introduced. Then clearly:

$$
\ln \hat{\phi}(a \xi)=\int_{0}^{a} \nu_{0}(u \xi) \frac{d u}{u}
$$

and

$$
\hat{\phi}(\xi)=\exp \left[\int_{0}^{1} \nu_{0}(u \xi) \frac{d u}{u}\right]
$$

Such a formula can be thought of as a continuous multiplicative formula, interpreting the exponential of the integral as a continuous product of exponentials. Indeed, setting

$$
\mu_{0}(\xi)=e^{\nu_{0}(\xi)}
$$

one can write the Fourier transform of the scaling function as:

$$
\hat{\phi}(\xi)=\prod_{u=0}^{1}\left(\frac{d u}{u}\right) \mu_{0}(u \xi)
$$

the continuous product $\prod_{u=0}^{1}\left(\frac{d u}{u}\right)$ with respect to the logarithmic measure $\frac{d u}{u}$ being defined by the right hand side of $(3-3)$. filters:

For $\xi$ such that $\hat{\phi}(a \xi) \neq 0$ introduce now the functions $m_{0}^{j}(\xi)$, called the integrated

$$
m_{0}^{j}(\xi)=\prod_{u=a_{j+1}}^{a_{j}}\left(\frac{d u}{u}\right) \mu_{0}(u \xi) .
$$

Such functions allow a discretization of the continuous product formula $(3-5)$, and make the connection with the structure appearing in the usual multiresolution context. The Fourier transform of the scaling functions can be written as:

$$
\hat{\phi}\left(a_{j} \xi\right)=m_{0}^{j}(\xi) \hat{\phi}\left(a_{j+1} \xi\right)
$$


Notice that this expression, together with the monotonicity of $|\hat{\phi}|$ can be used to define $m_{0}^{j}(\xi)$, according to:

$$
m_{0}^{j}(\xi)= \begin{cases}\frac{\hat{\phi}\left(a_{j} \xi\right)}{\hat{\phi}\left(a_{j+1} \xi\right)} & \forall \xi \text { s.t. } \hat{\phi}\left(a_{j+1} \xi\right) \neq 0 \\ 0 \quad \forall \xi \text { s.t. } \hat{\phi}\left(a_{j+1} \xi\right)=0\end{cases}
$$

Introducing now the high-pass filter:

$$
m_{1}^{j}(\xi)= \begin{cases}\frac{\hat{\Psi}^{j}(\xi)}{\hat{\phi}\left(a_{j+1} \xi\right)} & \forall \xi \text { s.t. } \hat{\phi}\left(a_{j+1} \xi\right) \neq 0 \\ 0 \quad \forall \xi \text { s.t. } \hat{\phi}\left(a_{j+1} \xi\right)=0\end{cases}
$$

an immediate consequence of $(2-9)$ is that the filters $m_{0}^{j}$ and $m_{1}^{j}$ fulfill the quadrature mirror filter condition, that is:

$$
\left|m_{0}^{j}(\xi)\right|^{2}+\left|m_{1}^{j}(\xi)\right|^{2}=1 \quad \forall j \in \mathbb{Z}, \quad \forall \xi \in \mathbb{R} \text { s.t. } \hat{\phi}\left(a_{j+1} \xi\right) \neq 0
$$

\section{3-2. Factorizable wavelet packets.}

We will call factorizable wavelet packets the wavelet packets generated by an infinitesimal wavelet $\psi$ and a decreasing sequence $\left\{a_{j}\right\}$ of positive real numbers with the property that there exists a positive real $\lambda>1$ such that $\forall j \in \mathbb{Z}$,

$$
a_{j+1}=\lambda^{-n(j)} a_{j}
$$

for some $n(j) \in \mathbb{N}^{*}$. In other words, factorizable wavelet packets correspond to sequences of scale parameters that are subsequences of geometric sequences. Introduce then the filter $m_{0}$ such that:

$$
\hat{\phi}(\lambda \xi)=m_{0}(\xi) \hat{\phi}(\xi)
$$

that is:

$$
m_{0}(\xi)=\prod_{u=1}^{\lambda}\left(\frac{d u}{u}\right) \mu_{0}(u \xi)=e^{\int_{1}^{\lambda} \nu_{0}(u \xi) \frac{d u}{u}}
$$

In that case, all the integrated filters $m_{0}^{j}$ factorize into products of dilated copies of $m_{0}$ :

$$
m_{0}^{j}(\xi)=\prod_{n=0}^{n(j)-1} m_{0}\left(\lambda^{n} a_{j} \xi\right) .
$$

This means that for numerical computations, the same filter can be used throughout the decomposition. It is then possible to build a algorithm for the computation of the wavelet packets coefficients with a pyramidal structure, as in the case of the QMF algorithm for the computation of the coefficients with respect to an orthonormal base of wavelets. Factorizable wavelet packets then seem more adapted for numerical implementation. This does not mean of course that non factorizable schemes are impossible to implement. There can be some specific problems (namely in acoustics and voice analysis, or in vision) for which the optimal paving of the Fourier space is 
fixed by some phenomenological results, and do not a priori correspond to factorizable schemes. Nevertheless, depending on the required precision, it is likely that such pavings can be approached by pavings corresponding to factorizable schemes.

Remark. In the particular case where $n(j)$ is constant (and can be set to 1 without loss of generality), all the wavelet packets are obtained by dilations of a unique function $\Psi$, and all the integrated filters are dilated copies of $m_{0}$ too. The previous discussion then leads to a fast (i.e. $N \ln N$ ) algorithm, with pyramidal structure, to compute continuous wavelet decompositions. Such an algorithm is in the same spirit than the "Algorithme à Trous" discussed in [Ho-KM-Mo-Tc], since it is obtained by replacing the initial infinitesimal wavelet by another wavelet for which one can use quadrature mirror filters. However, while the "Algorithme à Trous" is associated to an interpolation scheme, and then damages the localization of the wavelets in the Fourier space (see e.g. [Du]), the one proposed here uses the function $\Psi$ which has essentially the same decay properties than $\psi$ in the Fourier space. It then seems more adapted to signal analysis, at least for applications like those described in [Gr-KMMo]. Let us nevertheless stress that we have not proposed any discretization scheme for the (continuous) variations of the wavelet packets coefficient with respect to the translation parameter $b$. A brutal discretization of the translation parameter periodises the $m_{0}$ and then the $m_{1}$ filters, yielding a more usual QMF relation. However, the exact reconstruction property is then lost (notice that the errors can in general be controled).

\section{FACTORIZATION FORMULAS AND CONTRAST ANALYSIS.}

We now describe a multiplicative way of reconstructing a function $f \in L^{2}(\mathbb{R})$ from $s_{a}$ and the contrast functions, which can be introduced as $c_{a}=\frac{d_{a}}{s_{a}}$. A precise meaning can be given to the multiplicative reconstruction formula, by making some analyticity assumptions on the scaling function $\phi$, ensuring that the zeros of $s_{a}(x)$ are isolated (with respect to the dilation variable $a$, and for any $x \in \mathbb{R}$ ). We will discuss this analyticity property at the end of this section.

Let us first assume that $s_{a}$ is a real analytic function of $\left.a \in\right] 0, \infty\left[\right.$. Then, if $s_{u}(x)$ does not vanish for $u \in[a, A]$, one can write:

$$
s_{a}(x)=s_{A}(x) e^{-\int_{a}^{A} \partial_{u} \ln s_{u}(x) d u} .
$$

If now $\partial_{u} s_{u}(x)=-\frac{d_{u}(x)}{u}$ (which is right under the assumptions made on the scaling function $\phi$ ), one then has:

$$
s_{a}(x)=s_{A}(x) e^{\int_{a}^{A} \frac{d u(x)}{s_{u}(x)} \frac{d u}{u}} .
$$

Let us now make sense to the integral when $s_{u}(x)$ vanishes in $[a, A]$. The goal is that $(4-1)$ still holds in such a case. Let us assume that $a_{0}$ is the unique zero of $s_{u}(x)$ in the interval $[a, A]$, and set, for $\varepsilon>0$ :

$$
s_{a}(x)=\frac{s_{a}(x)}{s_{a_{0}-\varepsilon}(x)} \frac{s_{a_{0}-\varepsilon}(x)}{s_{a_{0}+\varepsilon}(x)} \frac{s_{a_{0}+\varepsilon}(x)}{s_{A}(x)} s_{A}(x)
$$




$$
=s_{A}(x) \exp \left[\left(\int_{a}^{a_{0}-\varepsilon} \frac{d u}{u}+\int_{a_{0}+\varepsilon}^{A} \frac{d u}{u}\right) \frac{d_{u}(x)}{s_{u}(x)}\right] \frac{s_{a_{0}-\varepsilon}(x)}{s_{a_{0}+\varepsilon}(x)} .
$$

Taking the limit $\varepsilon \rightarrow 0$, the analyticity of $s_{u}(x)$ implies the existence of $k \in I N$ and a function $h$ such that $s_{u}(x)=\left(u-a_{0}\right)^{k} h(u, x)$, with $h\left(a_{0}, x\right) \neq 0$. Then $\lim _{\varepsilon \rightarrow 0} \frac{s_{a_{0}-\varepsilon}(x)}{s_{a_{0}+\varepsilon}(x)}=$ $(-1)^{k}$ and $\frac{d_{u}(x)}{s_{u}(x)}=\frac{k}{u-a_{0}}+g(u, x)$, for some function $g$ continuous at $u=a_{0}$. Otherwise stated:

$$
s_{a}(x)=s_{A}(x) \exp \left[p . v \cdot \int_{a}^{A} \frac{d_{u}(x)}{s_{u}(x)} \frac{d u}{u}+k \pi i\right] .
$$

Notice that such a specification of the integral coincides with the one obtained by redefining the (real) integration interval $a \leq u \leq A$ by the union of the two intervals $a \leq u \leq a_{0}-\varepsilon, a_{0}-\varepsilon \leq u \leq A$, and the half-circle of radius $\varepsilon$ centered at $a_{0}$, and then taking the limit $\varepsilon \rightarrow 0$. Notice also that when $a_{0}=a$ or $a_{0}=A$, the integral diverges, which is compatible with $(4-1)$.

Let us denote by $\Xi$ the set of admissible infinitesimal wavelets such that in addition for any $f \in L^{2}(\mathbb{R}), s_{a}(x)$ is analytic with respect to $a$ for any $x \in \mathbb{R}$. One can then introduce the infinitesimal contrast function:

$$
\begin{gathered}
c_{a}(x)=-a \partial_{a} \ln s_{a}(x) \quad \forall a, x \text { s.t. } s_{a}(x) \neq 0 \\
=\frac{d_{a}(x)}{s_{a}(x)}
\end{gathered}
$$

that is essentially the details of $f(x)$ at the scale $a$ divided out by the approximation of $f(x)$ at the scale $a$ (this explains the name of contrast function). Then we have shown:

THEOREM 3.

Let $\psi \in \Xi$ be an infinitesimal wavelet, and let $f \in L^{2}(\mathbb{R})$. Then, for any $a<A$, one has:

$$
s_{a}(x)=s_{A}(x) \exp \left[\int_{a}^{A} c_{u}(x) \frac{d u}{u}\right]
$$

with the above specification of the integral.

$(4-4)$ can also be written as a multiplicative integral, or continuous product as follows:

$$
s_{a}(x)=s_{A}(x) \prod_{u=a}^{A}\left(\frac{d u}{u}\right) e^{c_{u}(x)}
$$

the continuous product being defined by $(2-5)$.

Remark. The factorization formula is independent of the determination of the complex logarithm between two singularities. It is then possible to specify a global determination of the logarithm for $a<u<A$, turning around the singularities.

Let us now come back to the question of the analyticity of $s_{a}(x)$. One has:

LEMMA. 

bound:

If $|\hat{\phi}(\xi)|^{2}$ is infinitely differentiable, such that moreover its derivatives satisfy the

$$
\left.\left|\partial_{\xi}^{k}\right| \hat{\phi}(\xi)\right|^{2} \mid \leq K C^{k} k !(1+|\xi|)^{-1-k} \quad \forall \xi \in \mathbb{R}, k \in \mathbb{N}
$$

for some constants $C, K>0$, then for any $f \in L^{2}(\mathbb{R}), s_{a}(x)$ is an analytic function of $a \in \mathbb{R}_{+}^{*}$ for all $x \in \mathbb{R}$. Moreover, $\partial_{a} s_{a}(x)=-\frac{1}{a} d_{a}(x)$.

Remark. Actually, such assumptions imply the strong (i.e. in norm) analyticity of the map $a \rightarrow|\hat{\phi}(a \xi)|^{2}$ with values in $L^{2}(\mathbb{R})$.

Proof of the lemma. Set $h(\xi)=|\hat{\phi}(\xi)|^{2}$. Let us estimate the remainder of the Taylor series of $s_{a}(x)$ around $a=a_{0}$.

$$
r_{n}=\frac{\left(a-a_{0}\right)^{n}}{n !} \partial_{a}\left[\int \hat{f}(\xi) h(a \xi) \xi^{n} d \xi\right]_{a=\tilde{a}_{0}}
$$

for $\tilde{a}_{0}$ between $a$ and $a_{0}$. By assumption, one can find some $\mu$ such that

$$
\int \sup _{\left|a-\tilde{a}_{0}\right|<\mu}\left|\hat{f}(\xi)\left(\partial^{n} h\right)(a \xi) \xi^{n}\right| d \xi<\infty
$$

so that the integral and the derivative can be permuted. Then

$$
\left|r_{n}\right| \leq\left|a-a_{0}\right|^{n} K C^{n} \int|\hat{f}(\xi)|\left(1+\left|\tilde{a}_{0} \xi\right|\right)^{-1-n} d \xi .
$$

Then $\lim _{n \rightarrow \infty} \sup _{\left|a-\tilde{a}_{0}\right|<\epsilon}\left|r_{n}\right|=0$ for $\left|a-a_{0}\right|<\epsilon$ and $\epsilon$ small enough, which proves the lemma.

It is worth noticing that contrast analysis becomes particularly simple in the case of the analysis of positive-valued functions, and positive-valued scaling functions (such as Gaussian functions for instance), as observed in [DD]. Indeed, in such cases one does not have to take care of the zeroes of the $s_{a}$ functions.

Let us finally briefly describe the discretization of the factorisation formula, more precisely its relationship to the wavelet packets we described in sections 2 and 3 . Consider again the strictly decreasing sequence $a_{j}$ of positive real numbers. Then for all integers $n>m$ the integral $\int_{a_{m}}^{a_{n}} c_{u}(x) \frac{d u}{u}$ can be truncated into integrals over smaller intervals, and the factorization becomes:

$$
s_{a_{n}}(x)=s_{a_{m}}(x) \prod_{j=m}^{n-1} \exp \left[\int_{a_{j+1}}^{a_{j}} c_{u}(x) \frac{d u}{u}\right]
$$

which reduces to the trivial expression

$$
s_{a_{n}}(x)=s_{a_{m}}(x) \prod_{j=m}^{n-1} \frac{s_{a_{j+1}}(x)}{s_{a_{j}}(x)} .
$$


Such a simple factorization can still be expressed in terms of integrated contrast coefficients. Indeed, introducing a family of wavelet packets such that $(2-3)$ holds, the associated integrated contrast coefficients can be defined as

$$
C_{j}(x)=\frac{D_{j}(x)}{s_{a_{j}}(x)}
$$

so that the factorization formula reads:

$$
s_{a_{n}}(x)=s_{a_{m}}(x) \prod_{j=m}^{n-1}\left(1+C_{j}(x)\right) .
$$

The contrast coefficients form a sufficient information for the characterisation of the analyzed function. The scheme defined by $(4-8)$ can very easily be used for numerical computations [DD],[Me-DD-Ge].

Remark. linear contrast analysis: Let us finally describe the linear analogue of the previous contrast analysis. Owing to Theorem 1', we introduce the contrast function:

$$
\chi_{a}(x)=\frac{\delta_{a}(x)}{\sigma_{a}(x)}
$$

Then, assuming that $\hat{\varphi}$ is of class $C^{\infty}$, such that in addition

$$
\left|\partial_{\xi}^{k}\right| \hat{\varphi}(\xi) \mid \leq K C^{k} k !(1+|\xi|)^{-1-k} \quad \forall \xi \in \mathbb{R}, k \in \mathbb{N}
$$

for some positive constants $C, K$, we obtain the analyticity of $\sigma_{a}(x)$ and:

$$
\sigma_{a}(x)=\sigma_{A}(x) \prod_{u=a}^{A}\left(\frac{d u}{u}\right) e^{\chi_{u}(x)}
$$

Such a continuous formula can also be discretized along the same lines than $(4-6)$.

\section{CONCLUSIONS.}

The continuous wavelet transform is naturally associated with an infinitesimal multiresolution scheme. The wavelet transform and the continuous set of approximations of the analyzed function are linked by some kind of scale derivative. This relationship is used to build a discrete set of wavelet packets by partial integration of the infinitesimal wavelet on scale intervals. Such wavelet packets allow exact reconstruction though the scale axis has been discretized. If the sequence of scale parameters involved is factorizable as a subsequence of a geometric one, the scale discretization scheme can be combined with a pyramidal time discretization scheme. One then loses the exact reconstruction property (notice that the corresponding discretization errors can probably be controled through appropriate frame estimates).

We have shown that this way of discretizing an infinitesimal multiresolution scheme on the scales uses the wavelet transform as a scale derivative. Moreover, it may be generalized to a logarithmic derivative. The resulting infinitesimal analysis is the "contrast function", and leads to a multiplicative reconstruction formula. Such 
a logarithmic way of performing multiscale analysis is in particular of great interest for the analysis of positive-valued signals, for which the troubles due to the zero-crossings are avoided. It may be extended directly to the 2-dimensional case, and applied to image analysis. It then provides a rigorous framework to associate two basic properties of human vision: multiscale information processing and logarithmic light sensitivity [Me-DD-Ge]. The discrete reconstruction scheme, based of partial integrations of contrast functions, gives rise to formulas that are almost equivalent to "ratios of low pass filters" (ROLPs) decompositions that have already proved their efficiency in image processing [To-Ru-Va].

\section{ACKNOWLEDGEMENTS.}

We are very indebted to $\mathrm{Ph}$. Tchamitchian for many enlightening discussions, and particularly for showing us that $p \in L^{1}(\mathbb{R})$. We thank D.J. Ripmeester for a careful reading of the manuscript. Thanks are also due to A. Grossmann and Y. Meyer.

M.A. Muschietti is grateful to CNRS, the Universities of Aix-Marseille I and III, and the University of Nancy for kind invitations.

This work was partially supported by the GDR "Ondelettes", CNRS.

\section{REFERENCES}

[Cal] A. Calderón, Intermediate spaces and interpolation, the complex method, Studia Math. 24 (1964) p. 113.

[Co-Me-Qu-Wi] R. Coifman, Y. Meyer, S. Quake, M.V. Wickerhauser, Signal processing and compression with wavelet packets, YALE University preprint (1990).

[Co-We] R. Coifman, G. Weiss, Extensions of Hardy spaces and their use in analysis, Bull. A.M.S. 83 (1977) p. 569.

[Co-Gr-Tc] J.M. Combes, A. Grossmann, Ph. Tchamitchian Eds, Wavelets, time-frequency analysis and phase space, Proceedings of an international conference, Marseille, IPTI (1989) Springer Verlag.

[Da1] I. Daubechies, The wavelet transform, time-frequency analysis, and signal analysis, IEEE Trans. Inf. Th. 36 (1990) p.961.

[Da2] I. Daubechies, Ten lectures on wavelets, NSF-CBMS conference, SIAM, to appear (1991).

[De-E-Gu-KM-Tc-To] N. Delprat, B. Escudié, P. Guillemain, R. Kronland-Martinet, Ph. Tchamitchian, B. Torrésani, Asymptotic wavelet and Gabor analysis: extraction of instantaneous frequencies, IEEE Trans. Inf. Th. 38 (1992) 644-664.

[Du] P. Dutilleux, An implementation of the "Algorithme à Trous" to compute the wavelet transform, in Wavelets, Time-Frequency methods and Phase Space, Combes \& Al. Eds., IPTI, Springer (1989) p.298.

[DD] M. Duval-Destin,Analyse spatio-temporelle de la simulation visuelle à l'aide de la transformée en ondelettes, Ph-D Thesis (1991).

[Fr-Ja-We] M. Frazier, B. Jawerth, G. Weiss, Littlewood-Paley theory and the study of function spaces, to appear in the CBMS-NSF Regional conference series.

[Ga] D. Gabor, Theory of communication, Journal of the I.E.E (London) 93 (1946) p.429.

[Fr-Mo] J. Froment, J.M. Morel, Analyse multiéchelle, vision stéréo et ondelettes, in Les ondelettes en 1989, P.G. Lemarié Ed., Lect. Notes in Math. (1990) Springer Verlag.

[Gr-KM-Mo] A. Grossmann, R. Kronland-Martinet, J. Morlet, Reading and understanding continuous wavelet transform, in Wavelets, Time-Frequency methods and Phase Space, Combes \& Al. Eds., IPTI, Springer (1989) p.2. 
[Gr-Mo] A. Grossmann, J. Morlet, Decomposition of Hardy functions into wavelets of constant shape, SIAM. J. Math. Anal. 15 (1984) p.783.

[Gui] A. Guichardet, Symmetric Hilbert spaces and related topics, Lect. Notes in Math. 261 (1972), Springer Verlag.

[He-Wa] C. Heil, D. Walnut, Continuous and discrete wavelet transform, SIAM Reviews 31 (1989) p.628.

[Ho] M. Holschneider, Inverse Radon transform through inverse wavelet transform, Preprint CPT-90/P2364, submitted to Inv. Prob.

[Ho-KM-Mo-Tc] M. Holschneider, R. Kronland-Martinet, J. Morlet, Ph. Tchamitchian, A real time algorithm for signal analysis with the help of the wavelet transform, in Wavelets, Time-Frequency methods and Phase Space, Combes \& Al. Eds., IPTI, Springer (1989) p.286.

[Ho-Tc] M. Holschneider, Ph. Tchamitchian, Pointwise analysis of Riemann's nondifferentiable function, Invent. Math. 105 (1991) 157.

[Ma] S. Mallat, Multiresolution approach to wavelets in computer vision, in Wavelets, Time-Frequency methods and Phase Space, Combes \& Al. Eds., IPTI, Springer (1989) p.298.

[Mar] D. Marr, Vision, Freeman \& Co. (1982).

[Me-DD-Ge] J.P. Menu, M. Duval-Destin, T. Gervais, Spatial Metric and image processing: an opportunity for advanced displays development, Proc. of the Conf. of the Society for Information Display, Las Vegas (USA), May 1990, p. 468.

[Me] Y. Meyer, Ondelettes et Opérateurs, I Ondelettes, Hermann (1989).

[Ru] W. Rudin, Real and Complex Analysis, 2nd edition, McGraw \& Hill Publ.

[Str] J.O. Stromberg, A modified Haar system and higher order spline systems, Conference in harmonic analysis in honor of Atoni Zygmund, II, p.475, W. Beckner \& Al Eds., Wadworth Math. Series.

[To-Ru-Va] A. Toet, L. Ruyven, J. Valeton, Merging thermal images by a contrast pyramid, Optical Engineering 28 (1989) p. 789.

[To1] B. Torrésani, Wavelets associated with representations of the affine WeylHeisenberg group, J. Math. Phys. 32 (1991) p.1273.

[To2] B. Torrésani, Time-frequency representations: wavelet packets and optimal decompositions, Ann. Inst. H. Poincaré, Physique Théorique 56 (1992) 215-234.

[Wi] M.V. Wickerhauser, INRIA lectures on wavelet packets algorithms, YALE University preprint (1991). 